\title{
Not all cardiac arrests are the same
}

\author{
Benjamin S. Abella MD MPhil
}

See related research article by Kutsogiannis and colleagues on page 1589 and at www.cmaj.ca/lookup/doi/10.1503/cmaj.100034

Competing interests: Benjamin Abella has received grants from Philips Healthcare, the Medtronic Foundation and the American Heart Association for research pertaining to the delivery of cardiopulmonary resuscitation and outcomes from cardiac arrest; he has also received honoraria from Medivance Corporation for lectures on therapeutic hypothermia.

This article was solicited and has not been peer reviewed.

Correspondence to: Dr. Benjamin S. Abella, benjamin.abella@uphs.upenn .edu

CMAJ 2011. DOI:10.1503 /cmaj.110982
I n-hospital cardiac arrest remains a major public health challenge. It affects more than 200000 people annually in the United States and Canada and has a survival-todischarge rate of less than $25 \% .^{1,2}$ Research on the topic has increased over the past decade, with investigations evaluating resuscitation quality, ${ }^{3}$ time to defibrillation ${ }^{4}$ and disparities in survival. ${ }^{5}$ Efforts to improve patient outcomes have included the development of better early warning systems to anticipate cardiac arrest ${ }^{6}$ and initiatives to strengthen the quality of resuscitation care itself. ${ }^{7}$ However, much remains to be learned about in-hospital cardiac arrest.

Cardiac arrests occurring outside of hospital represent a more uniform disease process from a pathophysiologic standpoint (most occur as a result of spontaneous cardiac arrhythmia in the setting of coronary artery disease) and have been more extensively studied than in-hospital arrests. In-hospital arrests represent a much more heterogeneous group of diseases, both with respect to the underlying medical condition of the patient, the cause of the arrest and the resuscitation environment (e.g., intensive care unit [ICU] v. general ward).

In the study by Kutsogiannis and colleagues reported in $C M A J,{ }^{8}$ the authors examined retrospective data from five years of in-hospital cardiac arrests occurring in four Canadian ICUs. The dataset includes survival rates at one and five years after arrest, an outcome measure not often included in studies of such events (most focus on survival to hospital discharge as the key outcome). Of 517 patients included in their study, Kutsogiannis and colleagues found that about $27 \%$ survived to hospital discharge and that about $24 \%$ of the original 517 were alive at one year and $16 \%$ at five years. Consistent with other investigations, the authors found that survival rates were highest

\section{- Ker pOINTS}

- In-hospital cardiac arrest is common and has a high mortality.

- Patients who survive to hospital discharge have a good prospect of longer-term survival.

- Survival depends more on the underlying pathophysiology than on the occurrence of the arrest per se. among patients with ventricular tachycardia or ventricular fibrillation as the initial arrest rhythm, as opposed to pulseless electrical activity or asystole. More prolonged resuscitation efforts were associated with poorer survival.

A number of key conclusions can be drawn from the work of Kutsogiannis and colleagues. A note of optimism can be found in the long-term survival data when examined somewhat differently from the authors' data presentation. Changing the denominator from the total number of patients who had an arrest to the number who survived to hospital discharge gives a survival rate of $91 \%$ at one year and $60 \%$ at five years. This suggests that, contrary to conventional wisdom, patients who experience in-hospital cardiac arrest have a real opportunity for long-term survival, if we can improve that key initial step: survival to hospital discharge.

With the advent of therapeutic hypothermia, a powerful treatment for patients initially resuscitated from cardiac arrest, such gains may be possible. ${ }^{9}$ Many patients in the cohort of Kutsogiannis and colleagues probably did not receive therapeutic hypothermia, given that the collection of data for the study ended in 2005 and meaningful uptake of this treatment modality occurred only in the past five years. Of course, neurologic status of survivors remains an important qualifier of this optimism and is not addressed by the current work. However, other large studies of out-of-hospital cardiac arrest have suggested that neurologic function of survivors can be very good, especially with the application of therapeutic hypothermia. ${ }^{10}$

A second and more scientifically intriguing conclusion can be considered based on the authors' ICU dataset. Consistent with findings from prior studies, survival varied considerably depending on initial rhythm, duration of cardiopulmonary resuscitation and severity of illness before the arrest (as measured by the APACHE II [Acute Physiology and Chronic Health Evaluation] score). Why does survival vary so much? A deceptively simple response is that not all cardiac arrests are the same. Although all in-hospital cardiac arrests have in common the abrupt and complete loss of cardiac output that can be remedied only by the restoration of cardiac activity, at a

All editorial matter in CMAJ represents the opinions of the authors and not necessarily those of the Canadian Medical Association. 
deeper level they do not represent the same underlying disease process. A patient who experiences ventricular fibrillation after a myocardial infarction is very different from the patient with sepsis who loses mechanical cardiac function from progressive acidosis and pulseless electrical activity. Yet, from a treatment standpoint (algorithms for advanced life support) and a statistical standpoint (investigations of survival from inhospital cardiac arrest), one generally considers these patients as having the same clinical state regardless of the underlying disease process.

Cardiac arrest more accurately represents the common pathway for a variety of critical illnesses. Patients whose pathophysiology allows for rapid reversal after in-hospital cardiac arrest, such as the patient with myocardial infarction mentioned earlier, have a strong potential for survival and should receive aggressive treatment, both during and after resuscitation. Others, such as the patient with sepsis and profound acidosis, have a much lower chance of survival, and consideration of aggressive treatment should be balanced with discussion of care goals with the family and consideration of care withdrawal.

The general pessimism toward survival after in-hospital cardiac arrest in our medical culture may rest with the mistaken opinion that the two patients described above have the same disease. Resuscitation guidelines attempt to separate these patients, in a sense, by virtue of classification of arrest by initial rhythm. This may tell only part of the story, because ventricular fibrillation can also occur in patients with end-stage sepsis, but it represents a different process.

In future studies, evaluation and treatment of cardiac arrest should become more patient-centred and grounded in physiology. To achieve these goals, future research should include the assessment of tools to evaluate patient physiology during cardiac arrest and resuscitation in real time. One size does not fit all with regard to treatment for these patients. If we can appropriately customize both evaluation and treatment, an important step forward may be gained in our attempts to improve patient survival after cardiac arrest.

\section{References}

1. Merchant RM, Yang L, Becker LB, et al. Incidence of treated cardiac arrest in hospitalized patients in the United States. Crit Care Med 2011; June 23 [Epub ahead of print].

2. Nadkarni VM, Larkin GL, Peberdy MA, et al. First documented rhythm and clinical outcome from in-hospital cardiac arrest among children and adults. JAMA 2006;295:50-7.

3. Abella BS, Alvarado JP, Myklebust H, et al. Quality of cardiopulmonary resuscitation during in-hospital cardiac arrest. JAMA 2005;293:305-10.

4. Chan PS, Nichol G, Krumholz HM, et al. Hospital variation in time to defibrillation after in-hospital cardiac arrest. Arch Intern Med 2009;169:1265-73.

5. Merchant RM, Becker LB, Yang F, et al. Hospital racial composition: a neglected factor in cardiac arrest survival disparities. Am Heart J 2011;161:705-11.

6. Kotsakis A, Lobos AT, Parshuram C, et al. Implementation of a multicenter rapid response system in pediatric academic hospitals is effective. Pediatrics 2011 Jun 20 [Epub ahead of print]

7. Edelson DP, Litzinger B, Arora V, et al. Improving in-hospital cardiac arrest process and outcomes with performance debriefing. Arch Intern Med 2008;168:1063-9.

8. Kutsogiannis DJ, Bagshaw SM, Laing B, et al. Predictors of survival after cardiac or respiratory arrest in critical care units. CMAJ 2011; Aug. 15 [Epub ahead of print].

9. Sagalyn E, Band RA, Gaieski DF, et al. Therapeutic hypothermia after cardiac arrest in clinical practice: review and compilation of recent experiences. Crit Care Med 2009;37:S223-6.

10. Cheung KW, Green RS, Magee KD. Systematic review of randomized controlled trials of therapeutic hypothermia as a neuroprotectant in post cardiac arrest patients. CJEM 2006;8:329-37.

Affiliation: Benjamin Abella is with the Center for Resuscitation Science and the Department of Emergency Medicine, University of Pennsylvania, Philadelphia, $\mathrm{Pa}$. 